\title{
The visual orientation memory of Drosophila requires Foraging (PKG) upstream of Ignorant (RSK2) in ring neurons of the central complex
}

\author{
Sara Kuntz, ${ }^{1}$ Burkhard Poeck, ${ }^{1}$ Marla B. Sokolowski, ${ }^{2}$ and Roland Strauss ${ }^{1,3}$ \\ ${ }^{1}$ Institut für Zoologie III-Neurobiologie, Johannes-Gutenberg Universität Mainz, D-55099 Mainz, Germany; \\ ${ }^{2}$ Department of Ecology and Evolutionary Biology, University of Toronto, Toronto, Ontario M5S 3B2, Canada
}

\begin{abstract}
Orientation and navigation in a complex environment requires path planning and recall to exert goal-driven behavior. Walking Drosophila flies possess a visual orientation memory for attractive targets which is localized in the central complex of the adult brain. Here we show that this type of working memory requires the cGMP-dependent protein kinase encoded by the foraging gene in just one type of ellipsoid-body ring neurons. Moreover, genetic and epistatic interaction studies provide evidence that Foraging functions upstream of the Ignorant Ribosomal-S6 Kinase 2, thus revealing a novel neuronal signaling pathway necessary for this type of memory in Drosophila.
\end{abstract}

[Supplemental material is available for this article.]

To analyze a visual orientation memory, we have developed the so-called detour paradigm in which flies walk on a circular, watersurrounded platform within a computerized cylindrical LED screen (Fig. 1; Neuser et al. 2008). In this assay, flies that are confronted with two opposing, inaccessible, and vertical dark stripes on the brightly illuminated cylinder will walk back and forth between the stripes for a considerable amount of time. When the fly is heading toward one stripe (Fig. 1A), thereby crossing the virtual midline of the platform, the stripes disappear and simultaneously a distracting stripe shows up laterally to the fly. The new target lures the fly out of its original pathway and it tries to approach the distracter (Fig. 1B). When the distracting stripe also disappears, the fly remembers the position of the original stripe and resumes walking into the direction of its original target (Fig. 1C).

Throughout this study 3- to 5-d-old flies, with their wings clipped under cold anesthesia $1 \mathrm{~d}$ prior to testing, were tested in the detour paradigm as described in Neuser et al. (2008). Ten approaches per fly were recorded and the percentage of choices toward the initial target was calculated for each fly (\% positive choices). Wild-type Canton-S (CS) flies recall the position of the initial target with a median frequency of $80 \%$ (Fig. 1D). Normality was tested with the Shapiro-Wilk Test. We used the Kruskal-Wallis Analysis of Variance Test for multiple comparisons and the Wilcoxon Matched Pairs Test for dependent samples in a one-by-one analysis. The nonparametric comparison against the random value was carried out with the one-sample Sign Test. The suitable test for a parametric comparison was the single $t$-test. Statistical analyses were performed with Statistica 7.0 and SPSS 20. See Supplemental Table S1 for all statistical calculations and numbers of flies tested.

Previous experiments with mutant flies that show structural defects in the brain revealed that an intact ellipsoid body, a part of the fly's central complex, is necessary for a functional orientation memory (Neuser et al. 2008). The central complex of Drosophila is a higher control center of locomotion and orientation

\footnotetext{
${ }^{3}$ Corresponding author

E-mail rstrauss@uni-mainz.de

Article is online at http://www.learnmem.org/cgi/doi/10.1101/lm.026369.112.
}

behavior and consists of four characteristic structures: the ellipsoid body (consisting of the typical ring neurons and radial elements connecting it to all other central complex neuropils), the fanshaped body, the protocerebral bridge, and the paired noduli (Strauss and Heisenberg 1993; Strauss 2002). On the other hand, flies of the learning mutant ignorant $\left(\mathrm{ign}^{58 / 1}\right)$ turned out to be defective in spatial orientation memory (Neuser et al. 2008). The ign gene encodes a protein kinase orthologous to the human Ribosomal-S6 Kinase 2 (RSK2) (Wassarman et al. 1994; Pereira et al. 2010), and mutants in this gene show deficits in associative and operant learning paradigms (Putz et al. 2004). ign ${ }^{58 / 1}$ is a null-allele and cell-type-specific cDNA rescue experiments have revealed that IGN is required in one or two specific subsets of ellipsoid-body ring neurons, R3 and/or R4d (after Renn et al. 1999), for a wild-type orientation memory (Neuser et al. 2008). So far IGN was the only protein known to be required for this type of working memory in flies. To get further insight into the signaling pathways required for the orientation memory, we aimed to identify other candidates and focused on one of the two cGMP-dependent protein kinases (PKG) known in Drosophila that are encoded by the foraging (for) gene (Kalderon and Rubin 1989). FOR was chosen because it is expressed in ellipsoid-body ring neurons (Belay et al. 2007) and for mutant larvae and flies have deficits in associative olfactory learning (Kaun et al. 2007; Mery et al. 2007). Two natural variants of the gene, the dominant allele called Rover $\left(f \circ r^{R}\right)$ and the recessive sitter allele $\left(f o r^{S}\right)$, are found in a relation of $70 \%-30 \%$ in natural populations. As the name implies, Rover larvae cover a significantly greater distance on a yeast plate when searching for food than sitter larvae (Sokolowski 1980). The two morphs differ by $10 \%$ in overall PKG activity as assessed in adult head extracts (Osborne et al. 1997; Fitzpatrick et al. 2007) and most likely will differ even more in FOR activity. Elevated levels of FOR are found in ring neurons and recently for has also been shown to be required in the central complex for visual pattern memory formation in flies (Wang et al. 2008). Together these findings prompted us to assess a possible function of for in orientation memory.

To investigate the role of FOR in visual orientation memory, we used the dominant $f o r^{R}$ allele, the recessive hypomorph $f o r^{S}$, 


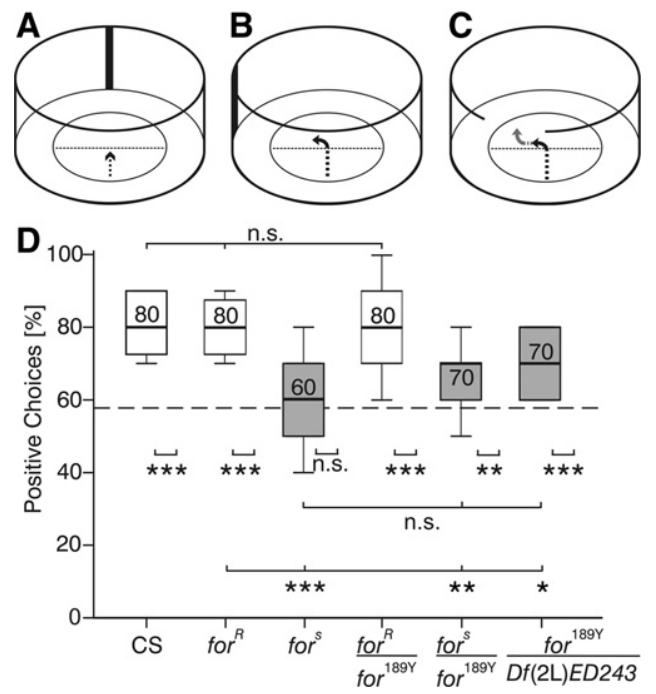

Figure 1. foraging mutants are defective in orientation memory as assessed by the detour paradigm. $(A-C)$ Schematic drawing of the detour paradigm. (A) A fly is heading for a vertical dark stripe at the opposite side of the arena. (B) When the fly crosses the virtual midline, the stripe disappears and simultaneously a distracting stripe appears laterally to the fly. The fly usually changes its path toward the distractor, which also disappears $1 \mathrm{sec}$ after the turnaround. (C) Left without a target, most wild-type flies turn back toward the position of the previous stripe. Such an orientation change is counted as a positive choice. (Method adapted from Neuser et al. 2008.) (D) Homozygous flies with the dominant $f \circ r^{R}$ allele behave like wild-type Canton-Special, whereas homozygous for and transheterozygous flies for the two hypomorphic alleles $\mathrm{for}^{\mathrm{s}} / \mathrm{for}^{189 \mathrm{r}}$ show no or a reduced memory performance, respectively. Combining the recessive for ${ }^{189 Y}$ allele with the $D f(2 L) E D 243$ deficiency deleting most of the for locus provided similar results, whereas heterozygous $\mathrm{for}^{R} / \mathrm{for}^{189 Y}$ are wild-type-like ( $n=24$ for all genotypes). Horizontal lines in the boxes represent the median frequencies of decisions for the old target, which are also indicated by numbers. The boxes denote the $25 \%$ and $75 \%$ quartiles, whiskers express $10 \%$ and $90 \%$ quantiles. The random decision level of $58 \%$ is marked by a dashed line (after a fly has turned to its right, there is a higher possibility for the following turn to be to the left side; $t$-test or Sign Test). (n.s.) Not significant; $\left(^{*}\right) P<$ $\left.0.05 ;{ }^{* *}\right) P<0.01 ;\left({ }^{* * *}\right) P<0.001$. Kruskal-Wallis Test for multiple comparisons; see Supplemental Table $S 1$ for detailed statistical analyses.

and another recessive allele ( for $^{189 Y}$ ) that was isolated in a P\{GAL4\} enhancer trap screen (Osborne et al. 1997; Wang et al. 2008). Homozygous Rover flies $\left(\right.$ for $^{R} /$ for $\left.^{R}\right)$ possess a wild-type orientation memory when compared to Canton-S controls (CS) (Fig. 1D). In contrast, homozygous sitter flies $\left(\right.$ for $^{s} /$ for $^{s}$ ) display no memory for the initial target, because their positive choices were not different from chance level. The chance level differs from $50 \%$ because a fly that has turned to the right is more prone to make a following left turn. Therefore, a preference index of $58 \%$ represents random behavior, i.e., loss of orientation memory (Neuser et al. 2008). The analysis of sitter mutants transheterozygous for the hypomorphic $f o r^{s}$ and $f o r^{189 Y}$ alleles revealed a considerable reduction of the orientation memory in the detour paradigm when compared to for $^{R} /$ for $^{R}$ flies (Fig. 1D). However, transheterozygous for $/$ for $^{189 Y}$ flies still possess a residual memory because their positive reactions for the initial target were significantly different from the $58 \%$ chance level. A similar result was obtained with flies transheterozygous for for ${ }^{189 Y}$ and a deficiency (Df(2L)ED243) (Tweedie et al. 2009) that specifically deletes a large part of the for gene locus. Moreover, heterozygous for $^{R} /$ for $^{189 Y}$ flies displayed wild-type behavior, corroborating the recessive nature of this for allele (Fig. 1D). Loss of orientation memory was also observed by inducing RNA inter- ference against the for transcription unit specifically in the ellipsoid-body ring neurons, further supporting the finding that FOR is required for visual orientation memory (see Supplemental Fig. S2).

Next we asked whether FOR function is needed in the same subtypes of ring neurons of the ellipsoid body as IGN. To address this, we performed tissue-specific cDNA rescue experiments in a $\mathrm{for}^{\mathrm{s}} / \mathrm{for}^{\mathrm{s}}$ mutant background using the UAS/GAL4-expression system (Brand and Perrimon 1993). Expression of FOR using the GAL4 driver line c232, which is specific for ring neuron types R3 and R4d (Renn et al. 1999), also restored the for mutant deficits to wild-type levels (Fig. 2A,D); this GAL4 line has been shown to rescue the orientation memory loss of the ign mutant (Neuser et al. 2008). To differentiate between the two sets of ring neurons targeted by the c232-GAL4 line, we made use of the 189Y-GAL4 line that expresses only in R3 ring neurons (Fig. 2C,D; Renn et al. 1999). Although this driver line represents a hypomorphic allele of the for gene (Fig. 1D; Osborne et al. 1997; Wang et al. 2008), the GAL4 expression pattern seems to only partially reflect the endogenous FOR pattern (Belay et al. 2007; Supplemental Fig. S1). A recent analysis of the transposon insertion site has shown that a P-element integrated into the lilli gene locus (Wang et al. 2008) at position 23C, whereas for localizes to 24A. Therefore, the GAL4 expression pattern might reflect the expression pattern of lilli. Nevertheless, 189Y-driven GAL4 and endogenous FOR are expressed in a similar pattern, including the ring neurons of the ellipsoid body and the $\alpha, \beta$, and $\gamma$ lobes of the mushroom bodies (Belay et al. 2007; Mery et al. 2007). In addition, 189Y induces expression of GAL4 in neurosecretory cells in the pars intercerebralis and in GABAergic interneurons of the antennal lobes (Lebestky et al. 2009; for a larger image of the section presented in Fig. 2C and a detailed expression analysis of the adult brain, see Supplemental Fig. S1). Indeed, expression of FOR using 189Y-GAL4 rescued the memory deficits of for $^{s} /$ for $^{189 Y}$ heterozygous mutants to wild-type levels, thus establishing that FOR is only necessary in the R3 neurons to restore visual orientation memory. As a control we used driver line 201Y-GAL4, which induces FOR in the same compartments (a- $/ \mathrm{b}-/ \gamma$-lobes) (Aso et al. 2009) of the mushroom body as 189Y-GAL4. This did not restore visual orientation memory, verifying that expression in the mushroom body did not play a role in the rescue with 189Y-GAL4. Next we asked whether the other types of ring neurons are also able to restore the for mutant orientation memory phenotype. However, using three additional driver lines to cover all other ring neurons (Renn et al. 1999) did not rescue the for phenotype (Fig. 2A). Therefore, FOR is only necessary and required in R3 neurons for the visual orientation memory.

The for gene is also expressed during development (Belay et al. 2007) and lethal alleles have been reported (Tweedie et al. 2009). To exclude the possibility that the reduced FOR function during development of the adult nervous system is negatively influencing adult orientation memory in $f o r^{s}$ mutant flies, we used an inducible expression system to confine FOR expression to the adult stage. The combination of the UAS-GAL4 system with the ubiquitously expressed, temperature-sensitive GAL4 repressor GAL80 $^{\text {ts }}$ (Tub $>$ GAL80 $^{\text {ts }}$ ) (McGuire et al. 2003) provides temporal control of FOR expression that can be induced by elevating the temperature. We therefore reared 189Y-GAL4-driven rescue flies at the restricted temperature $\left(18^{\circ} \mathrm{C}\right)$ and tested them for their orientation memory and, as expected, these flies show the memory defect (Fig. 2B). The flies were then shifted to $30^{\circ} \mathrm{C}$ overnight to induce additional FOR expression, tested again, and compared by a paired statistical analysis (Supplemental Table S1). This treatment rescued the memory deficits in for mutants, showing that additional expression of FOR in the adult ring neurons is sufficient to restore visual orientation memory (Fig. 2B). 


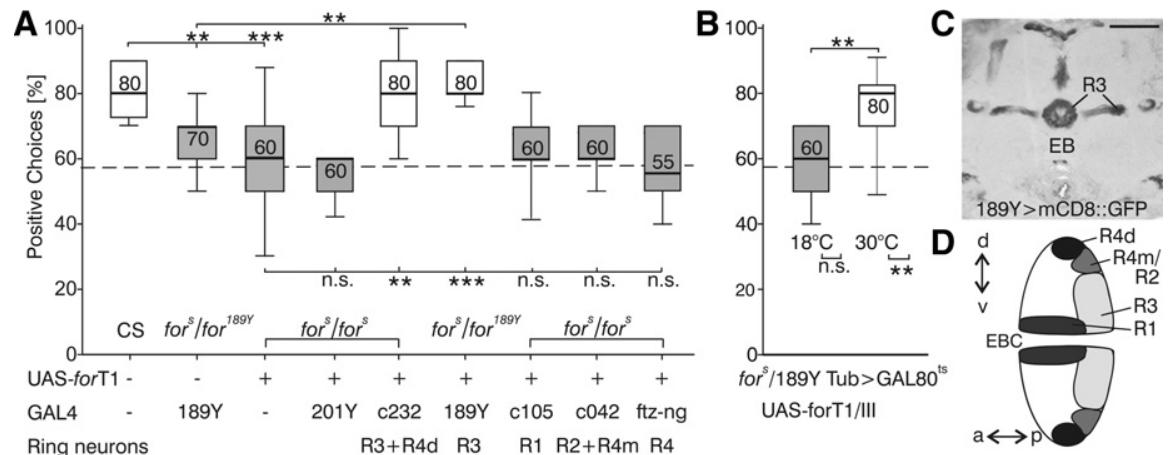

Figure 2. FOR is required in the R3 ring neurons of the adult ellipsoid body. ( $A$ ) Expressing a for CDNA (UAS-forT1) (Kaun et al. 2007) using the driver c232-GAL4 in ring neurons R3/R4d or using 189Y-GAL4 just in R3 can rescue the orientation memory deficit of for mutants. In contrast, control flies carrying just the UAS-forT1 ( $w / Y$; for ${ }^{s} /$ for $^{s}$; UAS-forT1/III) and flies expressing FOR in the mushroom bodies $(w / Y$; $\mathrm{for}^{\mathrm{s}} / \mathrm{for}^{\mathrm{s}} 201 \mathrm{Y}$; UAS-forT1/III) did not show amelioration of the for phenotype. Driver lines expressing in different types of ring neurons: c105-GAL4 (R1) (Renn et al. 1999), c42-GAL4 (R2/R4m) (Renn et al. 1999), and ftz-ng-GAL4 (R4) named ft2 in Pereanu et al. 2011) were also not able to restore the orientation memory. Male flies were tested $(n=20-24$; Kruskal-Wallis test for multiple comparisons; for $/ \mathrm{for}^{189 \mathrm{Y}}$ data identical with those presented in Fig. 1D). (B) $\mathrm{for}^{\mathrm{s}} / \mathrm{for}^{189 \mathrm{Y}} \mathrm{Tub}>\mathrm{GAL} 8 \mathrm{O}^{\mathrm{ts}}$; UAS-forT1/III rescue flies were reared at the restrictive temperature $\left(18^{\circ} \mathrm{C}\right.$ to prevent GAL4 activity) and tested in the detour paradigm. The same flies were retested on the next day after FOR expression was induced at $30^{\circ} \mathrm{C}$ overnight $(n=18$; Wilcoxon Matched Pairs Test and Sign Tests against $58 \%$ chance level; Supplemental Table S1). (C) The ellipsoid-body (EB) ring neurons type 3 (R3) are visualized via the membrane bound mCD8::GFP. Expression analysis of the 189Y-GAL4 driver line was done on 7- $\mu \mathrm{m}$ paraffin sections as described in Poeck et al. (2008) with a polyclonal anti-GFP rabbit serum (1:200 dilution; A11112 Invitrogen). Lines point to the lateral triangles, the dendritic field of the ring neurons (Renn et al. 1999), and their terminal field in the ellipsoid body. Scale bar, $50 \mu \mathrm{m}$. (D) Schematic drawing of a sagittal section through the midline of the ellipsoid body with the ring canal and the location of the four ring systems indicated anterior (a), dorsal (d), posterior ( $p)$, ventral $(v)$, ellipsoid-body canal (EBC). Location data adapted from Young and Armstrong (2010).

As mentioned above, like FOR, the IGN kinase is also required in the ring neurons of the adult brain for orientation memory (Neuser et al. 2008). To ascertain whether both genes act in the same genetic pathway, we conducted a genetic interaction study by reducing the gene copy number of ign in a hypomorphic for mutant background. Although transheterozygous for flies over a deficiency, and homozygous for $^{s} /$ for $^{s}$ display no orientation memory (Figs. 1D, 2A), transheterozygous for ${ }^{\mathrm{s}}$ / for ${ }^{189 Y}$ animals have an intermediate phenotype that is, however, still significantly different from wild-type and random levels (Fig. 1D). We therefore used this combination for genetic interaction studies because it allows us to detect suppressing as well as enhancing effects. Comparing for $^{\mathrm{s}} /$ for $^{189 Y}$ flies with flies that have in addition only one copy of the ign gene $\left(i g n^{58 / 1} /+;\right.$ for $^{s} /$ for $^{189 Y}$ ) (Fig. 3A) revealed a stronger memory deficit that was reduced to random levels. In contrast, removing one wild-type copy of the ign gene in for $^{R} /$ for $^{189 Y}$ heterozygous animals had no effect on the orientation memory (Fig. 3A). The genetic interaction of ign with the recessive for alleles suggests that both kinases act in the same signaling pathway and that both promote memory formation.

Having established a connection between the FOR and IGN kinases, we then aimed to elucidate the hierarchy of these two interacting proteins. We therefore investigated whether overexpression of
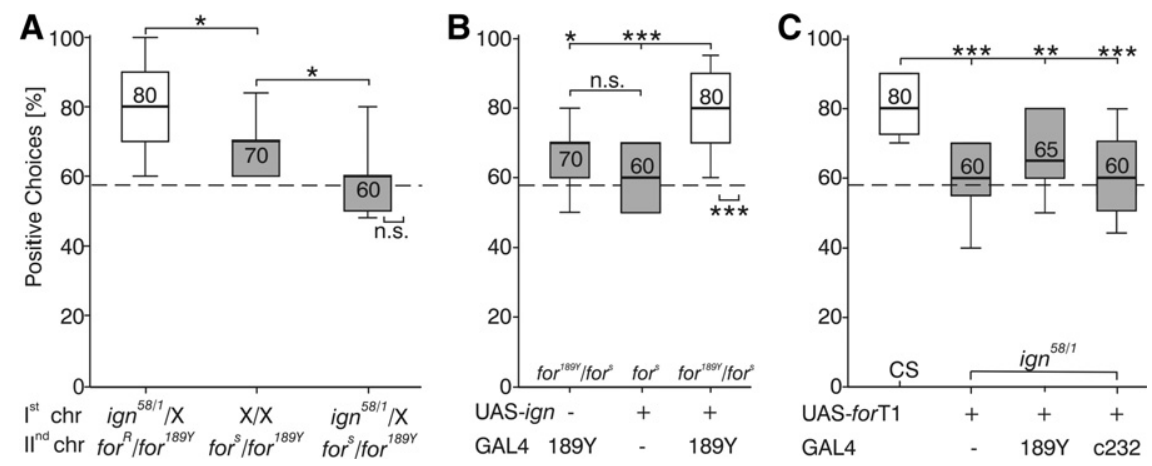

Figure 3. The FOR and IGN protein kinases share the same signaling pathway. $(A)$ Double heterozygous females for the two genes $\left(i g n^{58 / 1} / \mathrm{X} ; \mathrm{for}^{R} / \mathrm{for}^{189 \mathrm{Y}}\right.$ ) have a wild-type-like orientation memory, whereas $X / X ; f^{5} r^{5} / f^{189 Y}$ females displayed a reduced memory like males of the same genotype (cf. Fig. 1D). Reducing one copy of the ign gene enhanced this memory defect to chance levels $\left(i g n^{58 / 1}\right.$;

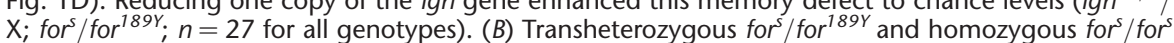
males carrying one UAS-ign transgene show reduced or no orientation memory, respectively. However, transheterozygous for $/$ for $^{189 Y}$-GAL4; UAS-ign/III can be rescued to wild-type levels. ( $n=24$ for all genotypes). (C) ign $^{58 / 1} / Y_{; j}$ UAS-forT1/III $(n=21)$ were analyzed in parallel with males that carried in addition either the for ${ }^{189}{ }^{\prime \prime}$-GAL4 or C232-GAL4 driver line $(n=24)$ and compared to wild-type controls $(n=24)$. Overexpression of the FOR kinase in R3 only or in R3 and R4d neurons was not able to rescue the reduced orientation memory of $i g n^{58 / 1}$ mutants. Kruskal-Wallis Tests for multiple comparisons and $t$-tests or Sign Tests against chance level (Supplemental Table S1). 
cerebellum (Feil et al. 2003). Furthermore, mice lacking PKG in the amygdala exhibit an impairment in fear conditioning (Paul et al. 2008) and cGMP/PKG signaling in the hippocampus is required for novel object recognition (Feil et al. 2009). In insects, FOR is involved in different types of food searching behavior and associative memories in which establishing the learning traces takes at least seconds (Reaume and Sokolowski 2009). In contrast, the orientation memory observed in the detour paradigm represents a form of working memory which has to be updated continuously in fractions of seconds. Whereas the phosphorylation and activation of FOR and IGN might be the mechanism by which these kinases affect longer-lasting memories, we think it is unlikely that this mechanism is involved in the constantly and rapidly changing orientation memory. Both kinases would have to be activated or inactivated in an online fashion during every turn of the fly. On the other hand, RSK2 has been implicated in multiple cellular processes and transcriptional control (Romeo et al. 2012). We therefore like to speculate that the biochemical pathway both kinases work in is necessary to endow the ring neurons with the capacity to efficiently change signaling rapidly to encode orientation. For instance, ring neurons might need a higher density of synaptic release sites and/or dendritic neurotransmitter receptors to exert their specific function.

\section{Acknowledgments}

The GAL4 driver lines c42, c105, 189Y, c232, and 201Y were kindly provided by D. Armstrong, and ftz-ng by J. Simpson. Wild-type Canton-Special, $D f(2 L) E D 243$, Tub-GAL80 ${ }^{\text {ts }}$, and UAS-mCD8:: GFP stocks were obtained from the Bloomington Stock Center. The UAS-forRNAi line was obtained from the VDRC (GD 38320) (Dietzl et al. 2007). We thank D. Kretzschmar for critical comments on the manuscript. This work was supported by the research fellowship Studienaufenthalte für Abschlussarbeiten of the German Academic Exchange Service (DAAD), by a Diploma and PhD scholarship of the Studienstiftung Rheinland-Pfalz, and by a grant from the Natural Sciences and Engineering Council of Canada.

\section{References}

Aso Y, Grübel K, Busch S, Friedrich AB, Siwanowicz I, Tanimoto H. 2009. The mushroom body of adult Drosophila characterized by GAL4 drivers. J Neurogenet 23: 156-172.

Belay AT, Scheiner R, So AK-C, Douglas SJ, Chakaborty-Chatterjee M, Levine JD, Sokolowski MB. 2007. The foraging gene of Drosophila melanogaster: Spatial-expression analysis and sucrose responsiveness. J Comp Neurol 504: 570-582.

Brand A, Perrimon N. 1993. Targeted gene expression as a means of altering cell fates and generating dominant phenotypes. Development 118: $401-415$.

Dietzl G, Chen D, Schnorrer F, Su KC, Barinova Y, Fellner M, Gasser B, Kinsey K, Oppel S, Scheiblauer S, et al. 2007. A genome-wide transgenic RNAi library for conditional gene inactivation in Drosophila. Nature 448: $151-156$.

Feil R, Hartmann J, Luo C, Wolfsgruber W, Schilling K, Feil S, Barski JJ, Meyer M, Konnerth A, De Zeeuw CI, et al. 2003. Impairment of LTD and cerebellar learning by Purkinje cell-specific ablation of cGMPdependent protein kinase I. J Cell Biol 163: 295-302.

Feil R, Hölter SM, Weindl K, Wurst W, Langmesser S, Gerling A, Feil S, Albrecht U. 2009. cGMP-dependent protein kinase I, the circadian clock, sleep and learning. Commun Integr Biol 2: 298-301.

Fitzpatrick MJ, Feder E, Rowe L, Sokolowski MB. 2007. Maintaining a behaviour polymorphism by frequency-dependent selection on a single gene. Nature 447: 210-212.

Gioia A, Zars T. 2009. Thermotolerance and place memory in adult Drosophila are independent of natural variation at the foraging locus. J Comp Physiol A Neuroethol Sens Neural Behav Physiol 195: 777-782.

Hawkins RD, Son H, Arancio O. 1998. Nitric oxide as a retrograde messenger during long-term potentiation in hippocampus. Prog Brain Res 118: $155-172$.
Hofmann F, Feil R, Kleppisch T, Schlossmann J. 2006. Function of cGMP-dependent protein kinases as revealed by gene deletion. Physiol Rev 86: 1-23.

Kalderon D, Rubin GM. 1989. cGMP-dependent protein kinase genes in Drosophila. J Biol Chem 264: 10738-10748.

Kaun KR, Hendel T, Gerber B, Sokolowski MB. 2007. Natural variation in Drosophila larval reward learning and memory due to a cGMP-dependent protein kinase. Learn Mem 14: 342-349.

Lebestky T, Chang J-SC, Dankert H, Zelnik L, Kim Y-C, Han K-A, Wolf FW, Perona P, Anderson DJ. 2009. Two different forms of arousal in Drosophila are oppositely regulated by the dopamine D1 receptor ortholog DopR via distinct neural circuits. Neuron 64: $522-536$.

McGuire SE, Le PT, Osborn AJ, Matsumoto K, Davis RL. 2003. Spatiotemporal rescue of memory dysfunction in Drosophila. Science 302: $1765-1768$.

Mery F, Belay AT, So AK-C, Sokolowski MB, Kawecki TJ. 2007. Natural polymorphism affecting learning and memory in Drosophila. Proc Natl Acad Sci 104: 13051-13055.

Neuser K, Triphan T, Mronz M, Poeck B, Strauss R. 2008. Analysis of a spatial orientation memory in Drosophila. Nature 453: 1244-1247.

Osborne KA, Robichon A, Burgess E, Butland S, Shaw RA, Coulthard A, Pereira HS, Greenspan RJ, Sokolowski MB. 1997. Natural behavior polymorphism due to a cGMP-dependent protein kinase of Drosophila. Science 277: 834-836.

Paul C, Schoberl F, Weinmeister P, Micale V, Wotjak CT, Hofmann F, Kleppisch T. 2008. Signaling through cGMP-dependent protein kinase I in the amygdala is critical for auditory-cued fear memory and long-term potentiation. J Neurosci 28: 14202-14212.

Pereanu W, Younossi-Hartenstein A, Lovick J, Spindler S, Hartenstein V. 2011. Lineage-based analysis of the development of the central complex of the Drosophila brain. J Comp Neurol 519: 661-689.

Pereira PM, Schneider A, Pannetier S, Heron D, Hanauer A. 2010. Coffin-Lowry syndrome. Eur J Hum Genet 18: 627-633.

Poeck B, Triphan T, Neuser K, Strauss R. 2008. Locomotor control by the central complex in Drosophila-An Analysis of the tay bridge mutant. Dev Neurobiol 68: 1046-1058.

Putz G, Bertolucci F, Raabe T, Zars T, Heisenberg M. 2004. The S6KII (rsk) gene of Drosophila melanogaster differentially affects an operant and a classical learning task. J Neurosci 24: 9745-9751.

Reaume CJ, Sokolowski MB. 2009. cGMP-dependent protein kinase as a modifier of behaviour. Handb Exp Pharmacol 191: 423-443.

Renn SCP, Armstrong JD, Yang M, Wang Z, An X, Kaiser K, Taghert PH. 1999. Genetic analysis of the Drosophila ellipsoid body neuropil: Organization and development of the central complex. J Neurobiol 41: $189-207$.

Romeo Y, Zhang X, Roux PP. 2012. Regulation and function of the RSK family of protein kinases. Biochem J 441: 553-569.

Sokolowski MB. 1980. Foraging strategies of Drosophila melanogaster-a chromosomal analysis. Behav Genet 10: 291-302.

Strauss R. 2002. The central complex and the genetic dissection of locomotor behaviour. Curr Opin Neurobiol 12: 633-638.

Strauss R, Heisenberg M. 1993. A higher control center of locomotor behavior in the Drosophila brain. J Neurosci 13: 1852-1861.

Taqatqeh F, Mergia E, Neitz A, Eysel UT, Koesling D, Mittmann T. 2009 More than a retrograde messenger: Nitric oxide needs two cGMP pathways to induce hippocampal long-term potentiation. J Neurosci 29: 9344-9350.

Tweedie S, Ashburner M, Falls K, Leyland P, McQuilton P, Marygold S, Millburn G, Osumi-Sutherland D, Schroeder A, Seal R, et al. 2009. FlyBase: Enhancing Drosophila Gene Ontology annotations. Nucl Acids Res 37: D555-D559.

Wang Z, Pan Y, Li W, Jiang H, Chatzimanolis L, Chang J, Gong Z, Liu L 2008. Visual pattern memory requires foraging function in the central complex of Drosophila. Learn Mem 15: 133-142.

Wassarman DA, Solomon NM, Rubin GM. 1994. The Drosophila melanogaster ribosomal S6 kinase II-encoding sequence. Gene 144: 309-310.

Young JM, Armstrong JD. 2010. Structure of the adult central complex in Drosophila: Organization of distinct neuronal subsets. J Comp Neurol 518: $1500-1524$

Zhuo M, Hu Y, Schultz C, Kandel ER, Hawkins RD. 1994. Role of guanylyl cyclase and cGMP-dependent protein kinase in long-term potentiation. Nature 368: 635-639.

Received March 12, 2012; accepted in revised form May 15, 2012. 


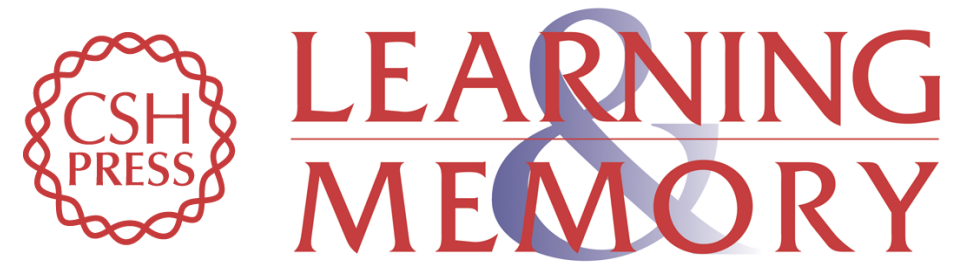

\section{The visual orientation memory of Drosophila requires Foraging (PKG) upstream of Ignorant (RSK2) in ring neurons of the central complex}

Sara Kuntz, Burkhard Poeck, Marla B. Sokolowski, et al.

Learn. Mem. 2012, 19:

Access the most recent version at doi:10.1101/lm.026369.112

Supplemental http://learnmem.cshlp.org/content/suppl/2012/07/18/19.8.337.DC1

Material

References This article cites 34 articles, 13 of which can be accessed free at: http://learnmem.cshlp.org/content/19/8/337.full.html\#ref-list-1

License

Email Alerting Receive free email alerts when new articles cite this article - sign up in the box at the Service top right corner of the article or click here. 